\title{
VIOLENCIA CONTRA LA MUJER EN EL PLANO JURÍDICO INTERNACIONAL Y COLOMBIANO
}

\author{
Alba Nidia Triana Ramírez
}

\section{Resumen}

El artículo trata, en primer lugar, acerca del panorama legislativo acordado en los organismos internacionales de Derechos Humanos en relación con el tema de la violencia contra la mujer. Para éste análisis se hace énfasis en los articulados que tratan la violencia doméstica en la relación de pareja. En segundo lugar, se establece la correlación entre los instrumentos jurídicos internacionales y la construcción de una normatividad en Colombia orientada a proteger los derechos fundamentales de las mujeres al interior de la familia. En tercer lugar, se hace un análisis a cerca del impacto de la normatividad en el ámbito de la realidad familiar colombiana en la actualidad.

Palabras clave: Derechos Humanos, violencia, mujer, instrumentos jurídicos internacionales, legislación colombiana.

\begin{abstract}
This article discuss about, in first place, concerning the legislative tune in international human right organisms in relation violence against woman's for this analysis we make emphasis in does articles that treat domestic violence in couple relationship. In second place, them selves establish a reciprocal way between international jurist instrument and a construction of a model in Colombia orientated to protect the fundamental rights of woman inside they family. In third place, we do analysis about the impact

Key words: Human Rights, violence, women, International Legal Documents, Colombian Legislation.
\end{abstract}

\section{Introducción}

La legislación constituye un marco normativo para la incorporación de la mujer a la sociedad y de la forma que asume su participación; sin embargo, el conjunto de leyes vigentes no necesariamente expresa el avance real de la incorporación de las mujeres a la 
vida social ${ }^{1}$

La segunda mitad del siglo XX se constituyó en escenario de grandes transformaciones sociales. Se destaca, entre otras, el reconocimiento de la mujer como sujeto social, político y de derecho. Asimismo, el tema de la discriminación y de la violencia contra la mujer, en los ámbitos social y familiar, se admitió como violación de derechos fundamentales.

Tales alcances se han dado de manera preponderante en el llamado "mundo occidental". Han sido, además, el resultado de la lucha, la reflexión y el debate público en la comunidad internacional y en los ámbitos nacionales.

En esta perspectiva, las diferentes convenciones, declaraciones y pactos internacionales de las Naciones Unidas (UN) y de la Organización de los Estados Americanos (OEA) -como resultado de las diferentes conferencias internacionales acerca de la mujer- llevaron a los Estados a consolidar legislaciones tendientes a la protección de los derechos fundamentales de la mujer. En este sentido, la discriminación y la violencia contra la mujer se constituyeron en una responsabilidad de los Estados y pasaron -en la década de los años noventa- a declararse como un problema de Derechos Humanos.

A través del presente escrito se pretende ofrecer un panorama de los factores que han permitido cambios en la legislación internacional y colombiana acerca del fenómeno de la violencia contra la mujer. Se pretende proporcionar argumentos para sondear la efectividad de la legislación frente al tema y brindar elementos para la comprensión, específicamente en la relación de pareja, en el contexto jurídico internacional y colombiano.

El análisis se sustenta en la legislación emitida por los organismos de Derechos Humanos: Naciones Unidas (UN), la Organización de los Estados Americanos (OEA), de las Conferencias Internacionales sobre mujer y de los recursos jurídicos colombianos, aprobados de conformidad con la reglamentación internacional.

1 Comisión Económica para América Latina y el Caribe, 1986: 51 
Violencia contra la mujer en el plano jurídico internacional y colombiano

Aunque se referencian algunos de los más importantes instrumentos jurídicos internacionales -la Declaración Universal de los Derechos Humanos, la Convención sobre los derechos políticos de la mujer (1952) la convención sobre la eliminación de todas las formas de discriminación contra la mujer (1979) y la Estrategia de Nairobi orientada hacia el futuro para el desarrollo de la mujer (1985) -el análisis se centra en la "Declaración sobre la eliminación de la violencia contra la mujer" de la Naciones Unidas (1993) y en la "Convención interamericana para prevenir, sancionar y erradicar la violencia contra la mujer" de la Organización de los Estados Americanos (1994). Se reconocen como los instrumentos fundamentales sobre los cuales se consolida la legislación colombina para la protección de los derechos fundamentales de la mujer.

En el caso particular de la legislación colombiana, se ha de recordar que durante la década de los años setenta del siglo pasado se establecen algunas iniciativas legislativas para la protección de los derechos fundamentales de la mujer; en especial, a partir de la reforma del Código Civil. Asimismo, con la Constitución Política Nacional de 1991 se abre la puerta para establecer una legislación propiamente dicha que proteja a las mujeres de la violencia domestica. Pero solo hasta 1996 aparece la primera ley colombiana para prevenir, remediar y sancionar la violencia intrafamiliar. Posteriormente, en el año 2000 se sanciona la primera legislación que se encarga de la protección, la promoción y la sanción sobre la violencia contra la mujer en la vida familiar.

Sin lugar a dudas, este proceso se constituye en el resultado de la plataforma jurídica y de los debates públicos que organismos internacionales consolidaron a partir de la década de los años ochenta del siglo XX.

\section{Mujer, violencia en la relación de pareja y legislación internacional}

Se torna paradójico ver cómo una situación vivida durante miles de años en el ámbito de lo privado llega al complejo mundo de lo público en tan solo medio siglo gracias a que a partir de 1946 surgen varios instrumentos significativos al respecto. En esa época se inicia un proceso de consolidación de la reglamentación jurídica internacional para la 
protección de los derechos fundamentales de la mujer y, en consecuencia, la situación de violencia que ha vivido la mujer al interior de la familia comienza a verse como un problema.

$\mathrm{Al}$ respecto, las diferentes conferencias internacionales que han tratado el tema de la condición de la mujer han jugado un papel importante al incorporar al debate público la situación de violencia al interior de la familia, situación que ha vivido y vive un inmenso número de mujeres en el mundo. Tales debates han permitido crear instrumentos jurídicos orientados a prevenir, sancionar y erradicar la violencia contra la mujer.

De ahí que sea pertinente, de acuerdo con los intereses del presente escrito, enunciar los principales órganos e instrumentos internacionales encargados de la protección de los derechos fundamentales de la mujer. Ahora bien, los avances efectuados en relación con la mejora de la condición social y jurídica de la mujer han sido realmente significativos entre 1946 y 2000.

En consecuencia, se reconoce cómo en 1946 las Naciones Unidas crean la Comisión Jurídica y Social de la Mujer, primer órgano concebido en pro de los derechos de la mujer. Los mandatos específicos de la Comisión se relacionan con el mejoramiento de las condiciones sociales y jurídicas de la mujer en relación con nacionalidad, raza, idioma y religión. De igual modo, dispone colocar a la mujer en un plano de igualdad con el hombre en todos los campos de la empresa humana. Se pretende así eliminar toda discriminación en lo relativo a derechos políticos, sociales y económicos existente en la legislación de los estados miembros.

Por su parte, la Resolución de la Asamblea General de las Naciones Unidas de 1952 trata de los derechos políticos de la mujer. El propósito fundamental se centra en los derechos al voto, a ocupar cargos públicos y a ejercer todas las funciones públicas establecidas por las legislaciones nacionales. Estos derechos ubican a las mujeres en el espacio de la vida pública, le otorgan la ciudadanía y establecen igualdad de condiciones políticas entre hombres y mujeres.

En el quinquenio 1975-1980 se proclaman derechos políticos en términos de participación y de asociación efectiva. De igual modo, se proclaman derechos de la familia 
Violencia contra la mujer en el plano jurídico internacional y colombiano

como la planificación familiar y se tocan problemáticas como la violencia doméstica. Sin embargo, el tema de la violencia contra la mujer en la familia no alcanza gran relevancia.

La Resolución 34/180 de 1979, emitida por la Asamblea General de las Naciones Unidas, se reconoce como una convención que pretende la eliminación de todas las formas de discriminación contra la mujer ${ }^{2}$. Admite que "las mujeres siguen siendo objetivo de importantes discriminaciones" y que tal discriminación "viola los derechos de la igualdad de derechos y del respeto de la dignidad humana" (Naciones Unidas, 1992: 166). Ratifica la igualdad del hombre y de la mujer en el goce de todos los derechos económicos, sociales, culturales, civiles y políticos:

Recordando que la discriminación contra la mujer viola los principios de igualdad de derechos y del respeto de la dignidad humana, que dificulta la participación de la mujer en la mismas condiciones que el hombre, en la vida política, social, económica y cultural de su país, que constituye un obstáculo para el aumento del bienestar de la sociedad y de la familia y que entorpece el pleno desarrollo de las posibilidades de la mujer para prestar servicio a su país y a la humanidad. (Naciones Unidas, 1979: 4)

De igual manera, la convención refiere que "para mejorar el estado de salud física y mental de todos los miembros de la sociedad se deben desarrollar políticas y programas encaminados a eliminar todas las formas de violencia contra la mujer y los niños" (Plata y Yanuzova, 1993: 24). Este hecho da inicio a una serie de conferencias y de sesiones que llevan a las Naciones Unidas a tomar como punto de referencia el tema de la violencia contra mujer en la familia. Así, el ámbito de lo privado empieza a tener cierta relevancia a nivel jurídico.

En la Segunda Conferencia, celebrada en Copenhague (Dinamarca, 1980), se aprueba por parte de las Naciones Unidas un programa de acción en favor de la mujer para la segunda mitad del decenio. Hace hincapié en la educación, el empleo y la salud.

${ }^{2}$ Convención. Acuerdo escrito concluido entre estados u otros sujetos de la sociedad internacional (las organizaciones internacionales, por ejemplo) con el objetivo de producir efectos de derecho en sus relaciones mutuas y que debe ser ejecutado de buena fe. En derecho francés, y según la constitución de la V República (1958), los tratados (firmados y ratificados) están por encima de las leyes. 
Promulga la resolución Quinta de Copenhague titulada "La mujer maltratada y la violencia de la familia". Documento que reconoce los malos tratos infligidos en la familia y la violencia doméstica contra la mujer; se les define como un problema complejo, con diversidad de causales que repercuten profundamente en el bienestar de la sociedad.

En la tercera Conferencia Mundial en 1985 se establece la "Estrategia de Nairobi", orientada hacia el futuro para el desarrollo de la mujer. Allí se instaura como prioritario la eliminación de la violencia en la familia y en contra la mujer: se consideran obstáculos básicos en la consecución de la igualdad, el desarrollo y la paz. De igual modo se insiste en el tema de la "victimización de la mujer", con miras a proteger a las mujeres contra comportamientos abusivos tolerados por distintas leyes en las prácticas tradicionales.

En el párrafo 288 de la "Estrategia de Nairobi" (literal E), respecto de "la mujer maltratada" puede leerse:

La violencia sexual va en aumento y los gobiernos deben afirmar la dignidad de la mujer, como cuestión de prioridad. Además los gobiernos deben intensificar para establecer o reforzar las formas de asistencia a las víctimas de este tipo de violencia, proporcionándoles alojamiento, apoyo y servicios jurídicos y de otra índole.

A partir de prestar asistencia inmediata a las víctimas de la violencia contra la mujer y la familia y la sociedad, los gobiernos deben tratar de crear convivencia pública sobre la violencia contra la mujer como un problema social, deben adoptar políticas y medidas legislativas para determinar las causas de esa violencia, impedirla y eliminarla, especialmente mediante la supresión de las imágenes y representaciones degradantes de la mujer en la sociedad y, por último, de propiciar el desarrollo de métodos de educación y reeducación destinados a los ofensores. (Naciones Unidas, 1985: 79).

Posteriormente, la Resolución de la Asamblea General de las Naciones Unidas del 20 de diciembre de 1993 establece, mediante una declaración, la eliminación de la violencia contra la mujer ${ }^{3}$. Por su parte, el vigésimo cuarto periodo ordinario de la Asamblea General

${ }^{3}$ Declaración. Texto solemne que proclama principios de suma importancia y de valor duradero. 
Violencia contra la mujer en el plano jurídico internacional y colombiano

de la Organización de los Estados Americanos (OEA) en 1994 aprueba la convención interamericana para prevenir, sancionar y erradicar la violencia contra la mujer.

Estos avances pueden considerarse limitados y circunscritos a un ámbito territorial determinado. Sin embargo, se ha de recordar que la protección universal de los derechos de las mujeres apenas sí había experimentado progresos significativos a mediados del siglo XX. La situación se torna relativamente comprensible en un contexto donde incluso resulta difícil situar la propia protección de la persona en el centro de la actividad jurídica en el ámbito internacional.

Solo hasta la década de los años noventa se reafirman los derechos de la mujer como derechos humanos decisivos para alcanzar condiciones mundiales de igualdad, desarrollo y paz ${ }^{4}$. Se les concede, desde entonces, un enfoque integral mucho más acertado y efectivo. De hecho, solo hasta la Cumbre de los Derechos Humanos de Viena (1993) se reconoce y proclama, de forma solemne, oficial y universal, la consideración de los derechos de las mujeres como derechos humanos.

A comienzos de años noventa se genera, entonces, una preocupación creciente por abordar el problema en todas sus formas. La violencia doméstica intrafamiliar se considera una problemática social íntimamente ligada a los derechos humanos. De ahí que sea importante resaltar las consideraciones efectuadas por las mujeres latinoamericanas en cuestión de sus derechos en la Reunión Satélite, "La nuestra", celebrada en San José de Costa Rica en diciembre de 1992.

La violencia contra las mujeres en sus múltiples manifestaciones constituye una violación sistemática en contra de los Derechos Humanos de 1948, frente a la cual no se han ofrecido mecanismos adecuados para visibilizarla, enfrentarla, prevenirla y garantizar

Aunque no tenga valor jurídico apremiante, puede ejercer una influencia como fuente de derecho reconocida unánimemente, como es el caso de la Declaración Universal de Derechos Humanos de 1948.

${ }^{4}$ Situación que se da gracias al despliegue de movimientos de mujeres que manifiestan su presencia en forma de ONGs en las cumbres internacionales. Cumbres celebradas desde principios de la década y en donde se tratan aspectos concretos: derechos humanos en general (Viena 1993), población y desarrollo (El Cairo, 1994). Tal vez la cumbre más importante en relación con el tema de mujer se adelanta en Beijing en 1995. 
acceso para denunciarla.

...La división establecida entre al ámbito privado y público, acentuada por organizaciones religiosas y culturales, han facilitado la impunidad sobre las diversas formas de violencia contra la mujer.

...En consecuencia, denunciamos como actos violatorios de nuestros derechos humanos, toda acción u omisión directa o indirecta, ya sea por parte del Estado o particulares, en el ámbito público o privado, infligido a las mujeres durante su vida, que tenga por objeto o por resultado causar sufrimiento físico, sexual, psicológico o emocional, dañar su integridad o su dignidad humana negando el derecho que tenemos a la autodeterminación en cualquier ámbito de nuestra vida, y cuya consecuencia sea menoscabar la seguridad en nuestra persona y nuestra autoestima, capacidades y personalidad.

"La Nuestra" busca un espacio en la legislación que considere la violencia contra la mujer en el ámbito público y privado: pretende que se pueda ocupar la protección de los derechos fundamentales de la mujer en términos de justicia, que se tome como una cuestión jurídica interna.

Esta lucha ha tenido una ardua trayectoria, cuyos resultados aún no han logrado establecerse en la realidad, aun cuando se han dado esfuerzos como los promulgados en la "Conferencia mundial del derechos humanos" (Viena, 1993), donde se subraya "la importancia de la labor destinada a eliminar la violencia contra la mujer en la vida pública y privada" (Naciones Unidas, 1993). En fin ausencia de resultados aun cuando el proceso desarrollado, tanto a nivel investigativo como de concientización, ha recorrido un complicado camino para que las organizaciones internacionales de protección a los derechos humanos, de derechos de la mujer y las Naciones Unidas reconozcan el problema de la violencia contra la mujer como un factor de derecho y de importancia.

La relación aquí expuesta quedaría incompleta si no se hace referencia a los instrumentos internacionales de mayor relevancia surgidos de la preocupación por el tema de la violencia contra las mujeres en su relación de pareja -violencia conyugal-. De manera particular se señalan la "Declaración sobre la eliminación de la violencia contra la mujer" y 
Violencia contra la mujer en el plano jurídico internacional y colombiano

la "Convención interamericana para prevenir, sancionar y erradicar la violencia contra la mujer”. Estos instrumentos definen y establecen criterios jurídicos en relación con el tema de la violencia contra la mujer al interior de la familia. Se constituyen, además, en referentes para Colombia en cuestiones de reforma y de estructuración de normas jurídicas internas a favor de la protección de los derechos fundamentales de la mujer.

1.1. Declaración sobre la eliminación de la violencia contra la mujer. En la octogésima quinta sesión plenaria de las Naciones Unidas (20 de diciembre de 1993) se aprueba, a partir del informe de la Tercera comisión (A/48/629), la Resolución 48/104 conocida como "Declaración sobre la eliminación de la violencia contra la mujer". Se convierte la Declaración en el primer instrumento de carácter jurídico internacional en relación con el tema de la violencia contra la mujer. Y aunque no tiene la fuerza de un tratado internacional, sí reúne requisitos de formalidad que promueven a los Estados Partes.

La Declaración reconoce que "la violencia contra la mujer constituye un obstáculo para el logro de la igualdad, el desarrollo y la paz" lo mismo que "para la aplicación de la Convención sobre la eliminación de todas las formas de discriminación contra la mujer" (Naciones Unidas, 1993).

Este último punto contribuye al desarrollo jurídico de las naciones: relaciona la violencia contra la mujer en el contexto de "los derechos y principios que están consagrados en los instrumentos internacionales: Declaración Universal de Derechos Humanos, el pacto internacional de derechos civiles y políticos, el pacto internacional de derechos económicos, sociales y culturales, la convención sobre la eliminación de todas las formas de discriminación contra la mujer" (Naciones Unidas, 1994). Pero tal vez lo más relevante de la Declaración se relaciona con la "Convención contra la tortura, y otros tratos o penas crueles, inhumanos o degradantes" (ib.), pues permite tipificar los diferentes tipos y formas de maltrato del cual son víctimas muchas mujeres del mundo en el ámbito privado: su familia. De ahí que otro punto de vital importancia logrado por la Declaración hace referencia a la interpretación dada a la violencia contra la mujer: no sólo se toma como un hecho público, también involucra la violencia privada como cuestión de violación de 
derechos humanos.

El artículo primero de la Declaración define la violencia contra la mujer como "todo acto de violencia basado en la pertenencia de sexo femenino que tenga o pueda tener como resultado un daño o sufrimiento físico, sexual o psicológico para la mujer, así como las amenazas de tales actos, la coacción o la privación" (ib.). De otro lado, la Declaración tipifica la violencia contra la mujer: causa de "daño o sufrimiento físico, sexual o psicológico".

Se ha de reconocer que estas y otras concepciones brindadas por la Declaración generaron argumentos de carácter jurídico que facilitaron el desarrollo de la legislación colombiana en relación con la problemática. Ofrecieron la oportunidad para que nuestra legislación interpretara y diera un manejo más apropiado a los problemas de violencia intrafamiliar, de modo específico aquellos relacionados con conflictos de pareja. Contribuyeron, además, al contenido en la legislación interna en dos aspectos. En primer lugar, llevaron a determinar de modo puntual los factores referidos con los términos sexual y psicológico. En segunda instancia, ayudaron a interpretar de diferente manera la concepción de lesiones físicas: contemplada como una más dentro del código penal colombiano sin definir claramente las relaciones que se dan con el agresor.

Ahora bien, gracias a la Declaración, la reforma de la legislación colombiana define la violencia contra la mujer no sólo como actos consumados; también tiene en cuenta las amenazas, la coacción y la privación arbitraria de la libertad. Aspectos que, en el caso de la violencia y el maltrato contra la mujer en la relación de pareja, han sido determinantes, pero de difícil interpretación por ocurrir entre individuos con cierta relación afectiva.

No obstante, lo más sobresaliente en la Declaración se da en la relevancia que da al espacio donde ocurre la agresión: por primera vez un instrumento jurídico internacional se ocupa del ámbito privado. Lo público, tanto en la legislación internacional como en la colombiana, había sido lo prioritario. De ahí que las leyes no aportaron los recursos adecuados para atender jurídicamente los casos de la violencia contra la mujer. A esta situación se le suma el hecho de que entra en juego lo sexual y lo psicológico, aspectos 
ligados a la privacidad de cada individuo.

Lo privado, el espacio de la familia, permaneció incuestionable por considerársele una institución autónoma y vital para el desarrollo de la sociedad. Al incursionar lo jurídico en el ámbito privado se genera debate público. De ahí que si se argumenta que de este modo se le están dando al Estado las herramientas jurídicas para que penetre y controle de manera pormenorizada a las personas. Se dirá, en consecuencia, que se estaría restando autonomía e individualidad: al comprometer el espacio privado quedaría absorbido lo particular a lo general. No obstante, existe otro punto de vista: frente a lo particular y colectivo, lo prevaleciente son los derechos fundamentales del individuo y, en este caso, los derechos de las mujeres.

El segundo artículo de la Declaración pone a la luz pública la violencia contra la mujer al interior de la familia al tratar de manera explícita situaciones como "los malos tratos", "la violación por el marido", "la violencia relacionada con la explotación" y "la violencia física, sexual y psicológica perpetrada o tolerada por el Estado donde quiera que ocurra". Elementos presentes históricamente al interior de la familia pero que factores culturales y sociales han mantenido ocultos, justamente bajo el argumento de que pertenecen al ámbito de lo privado.

Esta caracterización de violencia estuvo ausente en la legislación colombiana por largo tiempo: no se contemplaba ni como delito ni como argumento para la protección de la familia $y / o$ los derechos fundamentales de la mujer. La violación sexual, por ejemplo, era una figura no claramente interpretada en el ámbito jurídico nacional. Si se prestaba a ambigüedades jurídicas cuando se trata de un delito por fuera del grupo familiar, mucho más cuando se trataba de violación en la relación de pareja.

El Decreto-Ley 100 de 1980, Código Penal Colombiano, hizo evidente tal situación. De acuerdo con esa normatividad, conductas como el maltrato psicológico o sexual en la relación de pareja no se estipulan como delito específico contra la familia; hacían parte del ámbito general de las lesiones personales, siempre y cuando se hubiere producido una incapacidad médico-legal superior a 30 días. Las lesiones con incapacidad inferior se reducían a contravenciones. En consecuencia, la competencia para conocer de la 
denuncia no era de la autoridad jurisdiccional (el juez penal), sino de una autoridad administrativa (el inspector de policía).

Mas tarde, la Ley 360 de 1997 modifica algunas normas del titulo XI del libro II del Código Penal Colombiano relativos a los delitos contra la libertad y pudor sexual. El artículo 298 atañe al acceso carnal violento y el artículo 299 al acto sexual violento. Tales artículos establecen la penalización de estos delitos; sin embargo, no se hace ningún tipo de claridad respecto de la violación de la cual son victimas muchas mujeres por parte de sus maridos, especialmente en estado de embriaguez.

"La violencia relacionada con la explotación" se encontraba en iguales condiciones de las formas de violencia contra la mujer ya descritas. La legislación internacional, por ejemplo, juega con una connotación muy general pero no hace explícito qué debe interpretarse como "explotación". El término, en su condición práctica, hace referencia a la acción ocurrida en el ámbito público y queda el vacío interpretativo en relación con la aplicabilidad jurídica para su penalización en casos ocurridos al interior de la familia, en especial contra la mujer que, como se sabe, en países de América y África sufre tal condición en la cotidianidad de su vida familiar.

El literal C del segundo artículo de la Declaración que se ha venido analizando hace referencia a la responsabilidad del Estado frente al problema de violencia contra la mujer, "donde quiera que ocurra". De manera particular se hace referencia a la obligación que tienen los entes gubernamentales por velar y proteger los derechos de la mujer, más aún cuando los Estados pueden actualizar los recursos jurídicos internos a partir de los recursos internacionales ${ }^{5}$. Así pues, en términos generales, instrumentos jurídicos como tratados y convenios entre los Estados Partes de los organismos internacionales de derechos humanos como la ONU y la OEA han permitido fortalecer las leyes internas y han aportado

\footnotetext{
${ }^{5}$ Instrumentos jurídicos como "La convención sobre la eliminación de todas las formas de discriminación contra la mujer", "La Declaración sobre la eliminación de la violencia contra la mujer" y "La convención interamericana para prevenir, sancionar y erradicar la violencia contra la mujer" (OEA.), lo mismo que la "Estrategia de Nairobi orientada hacia el futuro para el adelanto de la mujer". Esta última presenta un diagnóstico muy completo de los grandes problemas de las mujeres y de la familia en el mundo y propone, además, unas estrategias puntuales que aportan a la estructuración de planes de desarrollo nacionales y locales.
} 
Violencia contra la mujer en el plano jurídico internacional y colombiano

a los planes de desarrollo argumentos válidos para prevenir, tratar y erradicar la violencia contra la mujer.

En esta línea, la comunidad internacional busca intervenir jurídicamente a través de los Estados en el espacio de lo privado: la familia. La Declaración reconoce la necesidad y la obligación que tienen los Estados para hacerle frente a la violencia contra la mujer. Se abre, entonces, la posibilidad de reconocer el problema y de formular alternativas de carácter social, socio-cultural y jurídico para mejorar las relaciones conyugales en la familia.

"No cabe duda de que la formación jurídica es el instrumento más apropiado para llegar al reconocimiento de los derechos humanos de las mujeres" (Plata y Yanuzova, 1993:14). Sin embargo, no basta con el conocimiento jurídico; éste debe ser el punto de partida para que en la realidad ocurra una verdadera transformación social. Transformación que beneficie tanto a mujeres como a hombres, niños y niñas, ancianos y a la familia y a la sociedad en general. De ahí que el reconocimiento jurídico se hace válido siempre y cuando se alcance su penetración en los ámbitos de lo cultural, lo social y lo político. Como afirma Estanislao Zuleta (1988:103), existe "un mínimo de condiciones que se pueden denominar como derechos humanos, pero el derecho no es más que un mínimo porque de nada sirven los derechos si no tenemos posibilidades".

El artículo cuarto de la Declaración es valioso en contenidos alternativos para la atención al problema. Allí se estipula claramente la obligación que tienen los Estados y las organizaciones, tanto gubernamentales como no gubernamentales, en la aplicación de medios apropiados para eliminar la violencia contra la mujer. Estas instituciones, según la Declaración, deben:

- Ratificar los instrumentos internacionales en pro de los derechos de la mujer.

- Abstenerse de practicar la violencia.

- Diligenciar legislativamente los actos de violencia.

- Establecer legislaciones nacionales, sancionatorias de todo acto de violencia contra la mujer. 
- Elaborar planes de acción nacional orientada a mantener y a proteger a la mujer contra la violencia.

- Prevenir jurídica, política, administrativa y culturalmente.

- Garantizar servicios de atención especializada.

- Asignar recursos.

- Hacer cumplir la ley (capacitar a funcionarios en el área).

- Educar.

- Investigar.

- Adoptar medidas para grupos vulnerables.

- Informar a las Naciones Unidas acerca de los problemas y de las medidas adoptadas para su atención.

Como puede observarse, el artículo cuarto define con claridad un plan de desarrollo que los Estados deben adoptar de acuerdo con las necesidades imperantes en cada país. En este orden de ideas, la Declaración contempla los lineamientos específicos para la construcción de un plan de acción orientado a tratar la violencia contra la mujer en los ámbitos público y privado. Plan ejecutable a partir de procesos como prevención, sanción, promoción, investigación y formación.

Por su parte, el artículo quinto compromete a las Naciones Unidas a contribuir “...en sus respectivas esferas de competencia, al reconocimiento y ejercicio de los Derechos y a la aplicación de los Derechos establecidos en la presente Declaración". Ratifica el compromiso de las Naciones Unidas en la promoción y protección de los derechos humanos y libertades fundamentales. Inquietud sustentada en la preocupación legítima de la comunidad internacional constatada en la Conferencia Mundial de los Derechos Humanos en Viena en 1993.

1.2. La convención interamericana para prevenir, sancionar y erradicar la violencia contra la mujer. Resolución aprobada por la Organización de los Estados Americanos (O.E.A.), a través de la Comisión Interamericana de Mujeres durante su vigésimo cuarto 
Violencia contra la mujer en el plano jurídico internacional y colombiano

período ordinario de las sesiones, en Belem Do Pará (Brasil) en 1994. Recoge las expectativas jurídicas en relación con la prevención, la sanción y la erradicación de la violencia contra la mujer. Se genera a partir de las conclusiones y recomendaciones de la Consulta Interamericana sobre la mujer, celebrada en 1990 y de la resolución AG/RES 1128 (XXI-0/91), llamada también "Sobre la erradicación de la violencia contra la mujer" adoptada por la vigésimo quinta Asamblea de delegados de la Comisión Interamericana de Mujeres.

La Convención puede catalogarse como un instrumento jurídico que compromete internamente a los Estados americanos. Su carácter decisivo exige respeto hacia los derechos de la mujer, pues es de índole obligatorio. Su objetivo consiste en determinar la violencia contra la mujer como una violación contra los derechos humanos y libertades fundamentales. Asimismo, pretende buscar mecanismos y alternativas desde los cuales se contribuya a la solución del problema de la violencia contra la mujer en todas sus formas.

En su preámbulo señala que la violencia contra la mujer representa una ofensa a la dignidad humana e inhibe el desarrollo individual y social y la plena e igualitaria participación de la mujer en todas las esferas de la vida, (OEA, 1994: preámbulo). Define la violencia contra la mujer "como cualquier acción conjunta basado en su género, que cause muerte, daño o sufrimiento físico, sexual o psicológico a la mujer tanto en el ámbito privado como público" (OEA, 1994: artículo primero).

Es muy importante la especificación que la Convención provee del acto de violencia al retomar el ámbito de lo privado. Al hablar del ámbito privado se refiere a los actos de violencia que particulares cometen contra las mujeres (por ejemplo la violencia conyugal). Se ha de entender, sin embargo, que el Estado se constituye en garante y responsable del bienestar de la familia y de los ciudadanos ${ }^{6}$. Por tanto, le compete la generación de políticas, planes y programas orientados al mejoramiento del nivel de vida de

\footnotetext{
${ }^{6}$ En el ámbito colombiano lo ratifica la Constitución Política Nacional de 1991 en el capítulo segundo del segundo título (De los derechos sociales, económicos y culturales). Allí dice: "la familia es el núcleo fundamental de la sociedad". Más adelante anota: "el Estado y la sociedad garantizan la protección integral de la familia". Artículo 42.
} 
la familia ${ }^{7}$. De igual manera, el Estado se encuentra obligado a la generación de leyes para la protección de los derechos y de las libertades fundamentales.

El artículo segundo de la Convención en el literal A hace una importante apreciación acerca del espacio en el cual se da este tipo de violencia y se refiere en primer lugar, de manera relevante, a la violencia que "tenga lugar dentro de la familia o unidad doméstica". De igual modo, se refiere de manera contundente a las relaciones interpersonales: "ya sea que el agresor comparta o haya compartido el mismo domicilio que la mujer, y que comprende entre otros violaciones y maltratos".

Es preciso aclarar que esta Convención es, quizá, el primer instrumento jurídico que en el ámbito regional define con claridad el problema de la violencia conyugal y la considera como acto punible. Así, pone en tela de juicio una problemática que se había gestado durante muchos años al interior de la familia sin que Estados, como el colombiano, se hubieran preocupado por adelantar la gestión jurídica para su definición y determinación.

El artículo cuarto de la Convención Interamericana se relaciona con los derechos protegidos; algunos de ellos coinciden con los estipulados en el artículo tercero de la Declaración. Sin embargo, aquél tiene en cuenta "el derecho a recurso sencillo y rápido ante los tribunales competentes que la amparen contra actos que violen sus derechos" (OEA, 1994: artículo cuarto). Además, ve la posibilidad de que la mujer víctima de la violencia o del maltrato conyugal pueda gestionar su problema ante los órganos judiciales competentes, de ahí su importancia.

En el caso de Colombia existían, al respecto, procedimientos inoperantes por cuanto impedían, siquiera, el inicio de un proceso que apuntara a la protección directa de las mujeres lesionadas en situaciones de violencia. Solamente con la promulgación del la Ley 294 de 1996 se pudo constituir una herramienta que pudiera atender jurídicamente esta situación, pues en su espíritu pretende prevenir, sancionar y erradicar la violencia al interior de la familia

"Así como la Declaración de los Derechos Humanos de las Naciones Unidas de

${ }^{7}$ Las relaciones entre Estado y familia en su proposición constitucional han resultado de la adopción de la "Declaración universal de los derechos humanos". 
Violencia contra la mujer en el plano jurídico internacional y colombiano

1948 estuvo precedida de las más diversas formas de violación y de desconocimiento de la persona humana..." (Umaña, 1995: 25), las circunstancias de discriminación y violencia que atentan contra la dignidad de las mujeres llevaron a la comunidad internacional a legislar para contrarrestar el fenómeno. Aún más, la presión de diversos grupos de mujeres organizadas en contra del desconocimiento y de la violación de los derechos fundamentales de la mujer, generados por actos de violencia, ha obligado a gestionar instrumentos jurídicos orientados a la eliminación de la violencia que padecen.

Por esta razón, el artículo octavo de la Convención Interamericana proporciona las herramientas para que los Estados Partes adopten de manera progresiva medidas específicas para prevenir, sancionar y erradicar la violencia contra la mujer. Dentro de los aspectos mencionados por la Convención se retoman para este análisis los referidos a los literales B, E y F. Las ideas allí expresadas poseen cierta relevancia en la generación de planes y programas para atender el problema de la violencia y maltrato contra la mujer en la relación de pareja ${ }^{8}$.

Modificar los patrones socioculturales de conducta de hombres y mujeres incluyendo el diseño de programas de educación formal y no formal apropiados a todo nivel del proceso educativo, para contrarrestar prejuicios y costumbres y todo otro tipo de prácticas que se basen en la primacía de la inferioridad o superioridad de cualquiera de los géneros o en los papeles estereotipados para el hombre y la mujer que la legitimizan o exacerban contra la mujer (OEA, 1993).

Lo cultural, en cuanto se refiere a costumbres, mitos y creencias acerca de la inferioridad por naturaleza o conveniencia de la sociedad, ha llevado a la mujer en convivencia conyugal al establecimiento de relaciones verticales de conminación y sometimiento por parte del hombre. Aunque hoy exista una profunda convicción de las capacidades intelectuales, laborales, sociales, políticas y de transformación cultural de las mujeres, el fenómeno de la discriminación y de la violencia continúan marcando las

${ }^{8}$ La Convención Interamericana para prevenir, sancionar y erradicar la violencia contra la mujer en el artículo octavo especifica y retoma algunos aspectos de la Declaración sobre la eliminación de la violencia contra la mujer en su artículo cuarto. 
diversas formas de relación entre los géneros. A esta condición ha contribuido y siguen contribuyendo ampliamente los procesos educativos y las diferentes prácticas pedagógicas. De ahí que el literal E plantee la necesidad de "fomentar y apoyar programas de educación gubernamentales y del sector privado destinados a concienciar al público sobre los problemas relacionados con la violencia contra la mujer, los recursos legales y la reparación que corresponda".

Este contenido compromete y motiva a los Estados y al sector privado para que se generen políticas educativas y legales que correspondan verdaderamente a una transformación social y al mejoramiento, por ende, de la familia. En ese sentido, el literal F especifica la necesidad de ofrecer "a la mujer objeto de la violencia acceso a programas eficaces de rehabilitación y capacitación que le permitan participar en la vida pública y privada".

A pesar de la magnitud del problema de violencia contra la mujer - en especial en la relación de pareja-, en la mayoría de los Estados latinoamericanos se carece tanto de políticas educativas que redunden en prevención como de programas de asistencia y rehabilitación. Tal aspecto se puede observar en el desenlace de diversos problemas sociales (maltrato, alcoholismo, drogadicción, abandono, etc.) que han marcado profundamente, y de manera especial, a las comunidades urbanas.

Finalmente, se puede observar que en la cuarta Conferencia Mundial sobre las Mujeres en Beijing también se definió la violencia contra la mujer. El documento refiere que la expresión "violencia contra la mujer" designa todo acto de violencia basado en el género que tiene como resultado posible o real un daño físico, sexual o psicológico, incluidas las amenazas, la coerción o la privación arbitraria de la libertad, ya sea que ocurra en la vida pública o en la privada.

Si bien es cierto, el tema de la violencia contra la mujer en el espacio doméstico ha sido reiterativo en todos las conferencias, reuniones y congresos de carácter regional y mundial, lo real consiste en que se llega al tercer milenio y este género continua, por lo menos en la mayoría de las países latinoamericanos, con una praxis precaria de la legislación que haga posible el respeto de sus derechos fundamentales humanos. Por tanto, 
Violencia contra la mujer en el plano jurídico internacional y colombiano

la lucha de las mujeres se debe promover en espacio serios y beligerante que obliguen a las sociedades a reconocer el poder que le ha arrebatado y que, como práctica didáctica, difundan los alcances que la Ley les ha otorgado.

Sin embargo, de nada sirve que las mujeres de este país conozcan sus derechos fundamentales si son cotidianamente víctimas de la violencia, maltrato y abandono por parte de sus esposos o compañeros; si laboralmente no tienen ninguna reivindicación; si se ven obligadas a responder en condiciones infrahumanas por su familia. En conclusión, si el sector de mujeres vulnerables a la violencia y a la discriminación es mayor que aquel que no lo es, cualquier implementación de normatividad es un arduo trabajo que compete a los diversos sectores involucrados.

\section{La violencia contra la mujer en la relación de pareja: el plano jurídico colombiano}

Los logros adquiridos por la mujer colombiana en materia jurídica son el producto de constantes luchas por salir del anonimato y declararse sujeto activo del derecho. Bajo la rotunda oposición de la clase política y de la iglesia, con unos medios de comunicación y el poder a favor de la estructura patriarcal, estos movimientos y manifestaciones femeninas se consagraron en una especial elite social, con relativo apoyo de las mujeres trabajadoras. Las clases populares tuvieron muy poca participación en esta primera etapa del proceso.

Sin embargo, esta fase histórica de la condición jurídica de la mujer, que indiscutiblemente avanzó en relación con sus derechos, no implicó cambios representativos en la realidad de la vida cotidiana del hogar. Son recursos jurídicos que tuvieron una basta resonancia en lo público, lógicamente importante para la sociedad, pero en el contexto de lo privado se la amordazaba con un código civil lesivo para el desarrollo personal ${ }^{9}$.

Antes de la reforma de 1974, algunos artículos del Código Civil muestran la

${ }^{9}$ El Código Civil Colombiano hasta 1991 se constituyó en el instrumento jurídico que trataba la situación de violencia intrafamiliar y contra la mujer como cualquier otra situación del contexto general. Una norma en defensa y sanción de la violencia intrafamiliar aparece solamente en 1996, con la Ley 294 la cual dictan normas para prevenir, remediar y sancionar la violencia intrafamiliar. 
sujeción de las mujeres al hombre en el espacio familiar; se constituye en una legislación violatoria y en la cual se le niega todo tipo de protección a sus derechos fundamentales. Esto a pesar de que Colombia era ya un estado partícipe de las Naciones Unidas, situación que obliga a la nación a reformar las leyes mediante las cuales se pusiera en entredicho la Declaración Universal de Derechos Humanos

A manera de ejemplo se exponen los siguientes artículos del Código Civil Colombiano (Plazas, 1962: 75) que pretenden subyugar la mujer al hombre y, además, se relacionan de manera estrecha con la familia:

-Artículo 176, inciso segundo: el marido debe protección a la mujer, y la mujer obediencia al marido.

-Artículo 177. La potestad marital es el conjunto de derechos que las leyes conceden al marido sobre la persona y bienes de la mujer.

-Artículo 179. El marido tiene derecho a obligar a su mujer a vivir con él y ella debe seguirle a donde quiera que traslade su residencia.

-Artículo 182. La mujer no puede, sin autorización del marido, celebrar contrato alguno, sin desistir de un contrato anterior, ni remitir una deuda, ni repudiar una donación, herencia o legado, etc.

-Artículo 195. Si la mujer casada ejerce públicamente una profesión o industria cualquiera (como la de directora de colegio, maestra de escuela, actriz, obstetriz, posadera, nodriza), se presume la autoridad general del marido para todos los actos y contratos concernientes a su profesión o industria, mientras no intervenga reclamación o protesta de su marido, notificada de antemano al público o especialmente al que contratare con la mujer.

Basta con leer cada uno de estos artículos para encontrar una estructura familiar jerarquizada, con una autoridad patriarcal que somete a la mujer en todos sus aspectos. Es notable en estas normas la ausencia total de la mujer en su propia reglamentación jurídica. Ausencia absorbida e interiorizada por una cultura que durante siglos había otorgado al hombre el poder de decisión sobre el sujeto femenino.

El hombre tenía el poder, en un contexto de relaciones desiguales, de legislar a su antojo sobre la mujer. Dado que ella durante largo tiempo no tuvo acceso al ámbito público 
Violencia contra la mujer en el plano jurídico internacional y colombiano

por considerársele inferior e incapaz, se vio sometida tanto al poder como a la fuerza masculina. Términos como "la mujer debe obediencia al marido", "el marido tiene derecho a obligar a la mujer", "la mujer no puede sin autorización del marido", etc. demuestran una profunda agresión jurídica hacia la mujer y anula cualquier posibilidad de ejercer derechos fundamentales: el derecho a la vida, igualdad ante la ley, al reconocimiento de la personalidad jurídica, a la libertad, a la dignidad, a la paz y la seguridad, entre otros.

Por esta razón, no es difícil sospechar que el problema de la violencia intrafamiliar, e incluso la violencia política y social de la cual es víctima actualmente la mujer colombiana, deba buscarse en sus raíces históricas. Pues se ha tenido como trayectoria un acumulado de factores culturales, en principio, y jurídicos, posteriormente, con los cuales se mantiene un esquema vertical en el grupo social primario.

La década de los años setenta se considera como trascendental para el desarrollo legislativo a favor de la mujer en Colombia. El decreto 1260 de 1970, estatuto del estado civil de las personas, suprimió la obligación para la mujer de usar el apellido del marido, precedido de a partícula “de”. Por su parte, el decreto 2820 de 1974 otorgó iguales derechos y obligaciones a las mujeres y a los varones y reforma algunos artículos del Código Civil colombiano relacionados con la familia.

Aunque con el decreto 2820 de 1974, sobre igualdad de derechos y obligaciones a las mujeres y a los varones, se otorgan mayores posibilidades a la condición jurídica de la mujer, sólo a través de la reforma a la constitución política colombiana de 1991 se proporcionan de manera abierta mayores garantías ${ }^{10}$.

La nueva constitución recoge algunos de los principios consagrados en la ley 51 de $1981^{11}$, y en el artículo decimotercero consagra el principio de igualdad de todas las personas ante la ley. Establece, además, en el inciso segundo la función del Estado que

${ }^{10}$ En Colombia rigió la Constitución de 1886 hasta 1991. En el transcurso histórico se dieron algunas reformas, pero muy poco se legisló a favor de la familia y específicamente a cerca de los derechos fundamentales de la mujer.

${ }^{11}$ Por medio de esta, el gobierno colombiano se suscribió a "La convención sobre la eliminación de todas las formas de discriminación contra la mujer", aprobada en 1979 por la Asamblea General de las Naciones Unidas y considerada la Declaración Internacional de los derechos de la Mujer. 
"promoverá las condiciones para que la igualdad sea real y efectiva y adoptará medidas en favor de grupos discriminados y marginados".

El artículo 43 de la Constitución Política Colombiana de 1991 refuerza de modo específico la condición de igualdad para la mujer: "La mujer y el hombre tienen iguales derechos y oportunidades. La mujer no podrá ser sometida a ninguna discriminación. Durante el embarazo y después del parto gozará de especial asistencia y protección del Estado, recibirá de éste subsidio alimentario, si entonces estuviese desempleada y desamparada. El Estado apoyará a la mujer cabeza de familia" (Constitución Política Nacional, 1991).

De acuerdo con el artículo 42, "las relaciones familiares se basan en la igualdad de derechos y deberes de la pareja y el respeto recíproco de todos sus integrantes. Cualquier forma de violencia se considera destructiva de su armonía y unidad y será sancionada conforme a la ley”. Sin embargo, solo hasta 1996, cuando se aprueba la Ley 294, se dictan normas para prevenir, remediar y sancionar la violencia intrafamiliar.

Así, por primera vez el tema de la violencia intrafamiliar en cualquiera de sus modalidades se constituye en una violación a los derechos fundamentales de la persona. El artículo tercero de dicha ley establece que para la interpretación y la aplicación de la misma se tendrán en cuenta principios que pueden ser aplicados a la protección de la mujer del maltrato de su pareja: primacía de los derechos fundamentales; toda forma de violencia en la familia se considera destructiva de su armonía y unidad y, por tanto, será prevenida, corregida y sancionada por las autoridades públicas; oportuna y eficaz protección especial a aquellas personas que en el contexto de una familia sean o puedan llegar a ser víctimas, en cualquier forma, de daño físico o psíquico, amenaza, maltrato, agravio, ofensa, tortura o ultraje, por causa del comportamiento de otro integrante de la unidad familiar; igualdad de derechos y oportunidades del hombre y la mujer.

La Ley 575 de 2000 modifica parcialmente la Ley 294 de 1996 y en el artículo cuarto establece:

Toda persona que dentro de su contexto familiar sea víctima de daño físico o psíquico, amenaza, agravio, ofensa o cualquier otra forma de agresión por parte 
Violencia contra la mujer en el plano jurídico internacional y colombiano

de otro miembro del grupo familiar, podrá pedir, sin perjuicio de las denuncias penales a que hubiere lugar, al Comisario de familia del lugar donde ocurrieren los hechos y a falta de éste al Juez Civil Municipal o promiscuo municipal, una medida de protección inmediata que ponga fin a la violencia, maltrató o agresión o evite que ésta se realice cuando fuere inminente.

Bajo la perspectiva de esta legislación, en Colombia se inicia una época importante en el marco de la promoción, la prevención, la sanción y la erradicación de la violencia intrafamiliar, y más específicamente sobre la violencia contra la mujer en la relación de pareja. Ahora bien, habrá que esperar qué efectos reales produce en la sociedad colombiana la Ley 575 de 2000, si contribuye a la práctica real de los derechos fundamentales de las mujeres, de acuerdo con lo establecido por la Carta Universal de los Derechos Humanos.

\section{La realidad colombiana frente a la violencia en la relación conyugal}

La revista Semana (2006-08-13) dio a conocer recientemente un caso de maltrato contra una mujer por parte de su marido en un prestigioso Club en la Ciudad de Barranquilla en Colombia. La nota tan solo representa cómo la violencia que mujeres de los diferentes estratos sociales de este país padecen cotidianamente:

En la última semana, como consecuencia del escándalo que produjeron los golpes que recibió de su marido una mujer en el Country Club de Barranquilla, los medios de comunicación, a partir de los relatos de las afectadas, se han ocupado de traer a la luz pública lo generalizada que es la violencia doméstica en todas las regiones y clases sociales [de Colombia]. Como reacción a esta nueva urgencia que ha ganado el problema, algunos han sugerido el endurecimiento de la legislación penal, tanto en el sentido de aumentar penas e impedir la excarcelación, como en el sentido de hacer que este delito pueda ser denunciado por cualquier persona, y no sólo por la víctima. Otros han sugerido que se haga énfasis en la prevención y la educación y no el uso de las herramientas legales.

En Colombia, al igual que en muchos otros países de América Latina, la mayor 
parte de los actos de violencia doméstica todavía se consideran como una cuestión privada. En consecuencia, no son denunciados, y no es posible conocer la verdadera magnitud del problema ${ }^{12}$. Según datos recibidos por el Alto Comisionado de los Derechos Humanos de Colombia, menos de la mitad de las mujeres golpeadas buscan ayuda, y sólo un nueve por ciento de las mismas formulan denuncias a las autoridades. La impunidad para los autores de hechos de violencia doméstica contra las mujeres es prácticamente del cien por ciento.

La violencia contra la mujer en la relación de pareja es el resultado del legado de la colonización: el poder jerarquizado en todos los ámbitos de la organización social, con un prototipo de modelo de familia basado en la representación de la Sagrada Familia, fundamento del catolicismo. Figura donde, por supuesto, la mujer no ocupó un lugar privilegiado; por el contrario, su papel se legitima en consonancia con el pensamiento judeocristiano, donde predominó la imagen patriarcal. Este hecho perdura con mayor fuerza en Colombia durante el régimen conservador ${ }^{13}$, pero se extiende hasta bien entrada la segunda mitad del siglo XX, y solo en la década de los años setenta, según se ha señalado, se hacen algunas transformaciones jurídicas a favor de la defensa de los derechos fundamentales de la mujer en el espacio doméstico.

Aunque con la penetración de las ideas liberales se presenta algunas reformas relacionadas con el matrimonio ${ }^{14}$, como contrato social, las instituciones siguieron reproduciendo relaciones de explotación y sometimiento amparadas en el lema de

\footnotetext{
${ }^{12}$ El propio Instituto de Medicina Legal de Colombia se refiere a un "subregistro" y aclara que las cifras no representan la totalidad de ese tipo de lesiones ocurridas en el país.

${ }^{13}$ Los regimenes Conservador y Liberal se constituyeron en los únicos partidos políticos que gobernaron Colombia a lo largo de la época republicana (1819 - 1988).

${ }^{14}$ El régimen Liberal sube al poder en 1930 y duró hasta 1946, luego asumen el poder nuevamente los conservadores y se extienden hasta 1957, incluyendo al régimen militar del General Rojas Pinilla. Entre 1958 y 1974, mediante un pacto entre liberales y conservadores, se establece el Frente Nacional. El pacto asociacionista del Frente Nacional en Colombia fue un acuerdo realizado en España por Alberto Lleras Camargo y Laureano Gómez (el pacto de Sitges, desarrollado en Benidorm, condenó la violencia, invitó a los colombianos a la paz y propuso una pausa, jurídicamente consagrada en la controversia de los partidos. Señaló el procedimiento del plebiscito para que el pueblo por sí mismo se diera una Constitución). Pero, además, tenía como objetivos hacerle frente a la dictadura del Gustavo Rojas Pinilla.
} 
Violencia contra la mujer en el plano jurídico internacional y colombiano

"fraternidad, libertad e igualdad". En función de ése lema, la legislación seguía imponiéndose a favor de la familia patriarcal.

Aunque en los últimos años el tema de mujer y desarrollo ha estado presente en la agenda de los gobiernos, falta una mayor atención al problema de la violencia intrafamiliar, en especial en lo relacionado con los factores jurídicos, educativos y de atención especial. Hoy, cuando ya ha transcurrido el primer quinquenio del siglo XXI, se podría decir que, en el ámbito público, la mujer ha avanzado en cuanto a la participación activa en el mercado laboral; pero, el poder, la dirección y la toma de decisiones, siguen siendo inequitativos, porque están concentrados en manos de los hombres. Sin embargo, en el ámbito privado (la familia) la situación no corresponde a los ingentes esfuerzos jurídicos desarrollados por los organismos internacionales. En América Latina, y especialmente en Colombia, por ejemplo, las relaciones de pareja no solamente siguen siendo desiguales respecto del empoderamiento, sino que subsiste un alto índice de violencia y maltrato contra la mujer. Así lo demuestran estudios como los presentados por Radio Universidad de Chile, en su diario electrónico del primero de diciembre del 2006: "en promedio, en Latinoamérica, entre un $30 \mathrm{y}$ un 45 por ciento de las mujeres han sido objeto de la violencia, ya sea física, sexual o psicológica". Este índice se sitúa en el 41 por ciento en Colombia, el 28 por ciento en Nicaragua, el 41 en Perú, el 27 en Haití, el 22 en la República Dominicana y el 40 por ciento en Chile y México, se eleva al 44 por ciento".

Para concluir, se hace alusión a las palabras del Alto Comisionado de la oficina en Colombia para los Derechos Humanos de las Naciones Unidas en la Audiencia Pública sobre víctimas de violencia intrafamiliar y abuso sexual (Bogotá, 30 de junio de 2005):

La República de Colombia se ha obligado solemnemente a adoptar, tanto en el ámbito de la legislación como en los campos administrativo y judicial, todas las medidas necesarias para que la mujer esté a salvo de cualquier forma de violencia intrafamiliar y de violencia sexual.

Colombia, es Estado Parte del Pacto Internacional de Derechos Civiles y Políticos aprobado por la Ley 74 de 1968; la Convención Americana sobre Derechos Humanos, aprobada por la Ley 16 de 1972; la Convención sobre la eliminación de todas las formas de 
discriminación contra la mujer, aprobada por la Ley 51 de 1981; la Convención para prevenir, sancionar y erradicar la violencia contra la mujer, aprobada por la Ley 248 de 1995; y la Convención Interamericana para prevenir, sancionar y erradicar la violencia contra la mujer, aprobada por la Ley 248 de 1995.

Sin embargo, después de nueve años de observar en el país la situación de los derechos humanos y del derecho internacional humanitario, la Oficina en Colombia del Alto Comisionado de las Naciones Unidas para los Derechos Humanos sigue estando preocupada por la especial situación de vulnerabilidad que afecta a las mujeres [...] frente a conductas antijurídicas con las cuales se lesionan o ponen en peligro sus derechos fundamentales a la vida, a la integridad personal, a la libertad individual y a la libertad, integridad y formación sexuales, o que afectan la armonía, la unidad y la honra de la familia. En desarrollo de sus actividades de observación, la Oficina sigue encontrando múltiples casos de violaciones de los derechos humanos e infracciones de la normativa humanitaria cuyas víctimas son mujeres y niños. Tal observación también ha recaído en conductas omisivas o negligentes de las autoridades nacionales con respecto a los perturbadores fenómenos de la violencia en el seno del grupo familiar y de la violencia materializada en violaciones, actos sexuales abusivos, prostitución forzada y esclavitud sexual. 
Violencia contra la mujer en el plano jurídico internacional y colombiano

\section{BIBLIOGRAFÍA}

Amnistía Internacional. (1995): Los derechos humanos: Un derecho de la mujer. Madrid: Amnistía Internacional (EDA).

Comisión Económica para América Latina y el Caribe. (1986): El decenio de la Mujer en el escenario latinoamericano: Realidades y perspectivas. Santiago de Chile: Naciones Unidas.

Naciones Unidas. (1948): Declaración Universal de los Derechos Humanos.

-(1979): Convención sobre la eliminación de todas las formas de discriminación de la mujer.

-(1985): Estrategia de Nairobi orientada hacia el futuro para el adelanto de la mujer. Conferencia mundial para el examen y la evaluación de los logros del decenio de las Naciones Unidas para la mujer: Igualdad, desarrollo y paz.

-(1993): Conferencia Mundial de Derechos Humanos. Declaración y programas de acción de Viena. Nueva York.

Naciones Unidas, Alto Comisionado para los Derechos Humanos de las Naciones Unidas, Oficina en Colombia. (2005): La violencia contra mujeres y niños: dificultades y retos. Bogotá.

Naciones Unidas, Asamblea General. (1993): Declaración sobre la eliminación de la violencia la mujer. Nueva York.

-(1994): Resolución 48/104. Nueva York

Naciones Unidas, Asamblea General, Comisión Económica para América Latina y el Caribe. División de Desarrollo Social Unidad Mujer y Desarrollo. (1992): Violencia Doméstica contra la Mujer. En: América Latina y el Caribe: Propuestas para la discusión serie Mujer y Desarrollo. Santiago de Chile.

Organización de los Estados Americanos, Comisión Interamericana de Mujeres. (994): Convención interamericana para prevenir, sancionar y erradicar la violencia contra la mujer. Convención de Belem Do Pará. Washington.

PLATA, M. I y YANUZOVA, M. (1993): Los derechos humanos y la convención sobre 
la eliminación de todas las formas de discriminación contra la mujer. Santafé de Bogotá: Profamilia-Servicio de Consultaría Jurídica Familiar.

PLAZA, A. (1962): Código Civil Colombiano. Bogotá: Voluntad.

Radio Universidad de Chile. Violencia doméstica,

$<$ http://www.radio.uchile.cl/notas.aspx?idnota=34599 >

República de Colombia. (1991) Constitución Política Nacional.

República de Colombia, Conserjería Presidencial para la Juventud, la Mujer y la Familia.

(1994): Segundo y tercer informe periódico de Colombia presentado al Comité para la eliminación de la discriminación contra la mujer. (CEDAW), Santafé de Bogotá.

República de Colombia, Plan Nacional de Rehabilitación. Programa de las Naciones Unidas para el Desarrollo (PNUD). (1994): Informe Nacional de Colombia preparado para la IV conferencia Mundial sobre la Mujer, Beijin,(China). Septiembre de 1995. Santafé de Bogotá.

UMAÑA LUNA, E. (1995): Estado - familia. Santafé de Bogotá: Universidad Nacional de Colombia: Facultad de Derecho, Ciencias Políticas y Sociales.

ZULETA, E. (1994): Elogio a la dificultad y otros ensayos. Santafé de Bogotá: Fundación Estanislao Zuleta.

—(1988): Participación y democracia en Colombia. En: Revista Foro, n ${ }^{\circ} 6$. 